\title{
Estrategia metodológica para fortalecer e incentivar habilidades de liderazgo en los escolares de nivel primario
}

\author{
Methodological strategy to strengthen and encourage leadership skills in \\ primary school students
}

\author{
Braulio Iván Tacagua Chirinos \\ ivankupidos@gmail.com \\ Universidad Autónoma Tomas Frías Potosí - Bolivia
}

\section{RESUMEN}

El presente artículo presenta los aportes más significativos del trabajo de investigación en torno a las estrategias metodológicas para fortalecer e incentivar habilidades de liderazgo en los escolares de nivel primario de la unidad educativa Domingo Faustino Sarmiento de la localidad de Yura. El objetivo de la investigación realizada fue, el diseñar de manera clara y precisa una estrategia metodológica que vaya acorde a sus edades y también a sus necesidades para las habilidades de liderazgo en el proceso formativo de los escolares en los cursos de cuarto, quinto y sexto de primaria. La metodología empleada fue: Histórico-lógico y de análisis-síntesis, realizando las observaciones necesarias para el análisis de datos y utilizando también cuestionarios entrevistas y una guía de observación. El presente artículo, ofrecerá la propuesta de una estrategia pedagógica de formación de líderes, que permita generar procesos acordes a los cambios tecnológicos, afín a la diversidad cultural para distinguir los problemas frente a oportunidades. Se puede concluir que la propuesta realizada, podrá ayudara a que los estudiantes adquieran el abordaje del tema tanto en la parte teórica como en la práctica.

Palabra clave: Estrategia metodológica; habilidades de liderazgo; escolares de primaria; unidad educativa Domingo Faustino Sarmiento

\section{ABSTRACT}

This article presents the most significant contributions of research work on methodological strategies to strengthen and encourage leadership skills in primary school students of the Domingo Faustino Sarmiento educational unit in the town of Yura. The objective of the investigation was to design a clear and precise methodological strategy that matches their ages and also their needs for leadership skills in the training process of schoolchildren in the fourth, fifth and sixth courses from elementary school. The methodology used was: Historical-logical and analysis-synthesis, making the necessary observations for data analysis and also using interview questionnaires and an observation guide. This article will offer the proposal of a pedagogical strategy for the formation of leaders, which allows generating processes according to technological changes, related to cultural diversity to distinguish problems from opportunities. It can be concluded that the proposal made may help students acquire the approach to the subject both in theory and in practice.

Key words: Methodological strategy; leadership skills; elementary school children; educational unit Domingo Faustino Sarmiento 
INTRODUCCIÓN

Las personas poseen aptitudes, habilidades y conocimientos, que los ayuda a incorporarse a la sociedad, en la mayoría de los casos las personas no aprovechan las potencialidad que tienen, y por ende no se muestra el liderazgo que requiere encaminar todo proceso educativo. Se podría decir, que el liderazgo y el proceso formativo son utilizados de manera errónea, porque no permite obtener una participación activa y consciente del estudiante como persona en formación.

Según Rojas y Gaspar (2006) en la actualidad se puede evidenciar que las personas viven con cualidades naturales, mismas que no están siendo bien aprovechadas, estas cualidades se manifiestan desde espacios escolares y estos espacios no tienen áreas para desarrollar el liderazgo en las actividades que se realizan a diario. Se hace necesario que la escuela genere procesos educativos y espacios educativos que vayan encaminados al desarrollo integral de las personas y al desarrollo del liderazgo. La educación debe promover la formación de líderes.

Se puede percibir que la formación educativa está centrado en los conocimientos y saberes, el maestro está ocupado en culminar su plan de clase y no así en generar líderes activos, y por otra parte el estudiante solo se preocupa por saber los diferentes contenidos en las diferentes áreas del conocimiento, por tanto el niño/niña está lejos de ser un emprendedor, creador, innovador, es decir, hace falta la formación integral de las personas.

El líder es un ciudadano de bien, que posee una educación integral con visión de futuro. El ser líder es una función esencial que depende fuertemente de la comunicación y la formación. El líder tiene que tener la capacidad de poder solucionar los problemas para bien, realizar actividades en las cuales sus decisiones no solo se basen en el mismo, si no, en un proceso formativo con interés en todas las personas; en bien de cada uno y en el bien común. (Coleman, 2012).

El sistema educativo nacional se enfrenta al desafío de las nuevas generaciones, es decir, el surgimiento de un nuevo tipo de estudiante, nacido y educado bajo la era de la tecnología, lo que obliga a la reformulación del proceso de aprendizaje, debido a que los escolares se educan con diferente visión y por ello es importante y necesario educar en valores y generar líderes para estos tiempos con propósitos y metas para luchar por el bien común y el de su lugar de origen. Así se hace imperioso dar respuesta a la interrogante: ¿De qué manera fortalecer las habilidades de liderazgo en el proceso formativo de las estudiantes de nivel primario en la unidad Educativa Domingo Faustino Sarmiento de la localidad de Yura?

Lo anterior permitió formular como objetivo de la investigación que dio las bases para este artículo, el diseñar de manera clara y precisa una estrategia metodológica acorde a sus edades y también a sus necesidades y contexto para las habilidades de liderazgo en el proceso formativo de los/las escolares de la escuela Domingo Faustino Sarmiento de la localidad de Yura, en los cursos de cuarto, quinto y sexto de primaria.

Asimismo, se buscó dar respuestas a las siguientes interrogantes relacionadas con el fundamento teórico que respaldara el proceso de la formación de líderes en los niños/as de la escuela domingo Faustino Sarmiento de la localidad de Yura a determinar las habilidades y destrezas de liderazgo en el proceso formativo de los/las escolares de la escuela Domingo Faustino Sarmiento de la localidad de Yura, en los cursos cuarto, quinto y sexto de primaria, además de analizar los elementos fundamentales de las estrategias 
metodológicas para el fortalecimiento de las habilidades de liderazgo en el proceso formativo de los/las escolares de la escuela Domingo Faustino Sarmiento de la localidad de Yura.

\section{MÉTODO}

En atención a lo expuesto al respecto por Sampieri (2010) el diseño metodológico de la investigación fue de carácter propositivo porque invitó a los escolares a ser líderes, y de tipo descriptivo debido a que en primera instancia permitió conocer a los escolares para tomar decisiones; lo que llevó a la realización de conceptos característicos de los procesos formativos de líderes en la escuela Domingo Faustino Sarmiento de la localidad de Yura. Concretando la metodología de la investigación se utilizaron los métodos: histórico-lógico y el de análisis-síntesis; el primero permitió buscar información y analizar el proceso de cambio que ha sufrido el objeto de estudio para la comprensión de la realidad desde la perspectiva histórica hasta la actualidad. El segundo condujo los pensamientos, empezando por los objetos más sencillos más fáciles de conocer, para subir gradualmente hasta el conocimiento más complejo en la elaboración y construcción del marco teórico, misma que permitirán la fundamentación teórica y conceptual a partir del objeto de estudio.

Con respecto a las técnicas $\mathrm{e}$ instrumentos de investigación se utilizaron: los cuestionarios en las/los escolares de la escuela Domingo Faustino Sarmiento de la localidad de Yura", para obtener datos importantes y tomar decisiones favorables que fortalezcan en su liderazgo; la entrevista para recolectar datos en la indagación general a los docentes de la escuela Domingo Faustino Sarmiento de la localidad de Yura, para obtener más información sobre la formación de líderes y por último la guía de observación para llegar a una conclusión general para la toma de decisiones.

\section{RESULTADOS Y DISCUSIÓN}

A continuación se describen la propuesta acerca de la estrategia metodológica con un enfoque sociocomunitario productivo acerca de las habilidades que deben adherirse al liderazgo durante el proceso de enseñanza y aprendizaje de los estudiantes.

Entendiéndose como liderazgo a la influencia hacia los demás, supone lograr un cambio de actitud o de conducta principio fundamental del liderazgo puesto que las personas tienden a seguir a quienes, desde su punto de vista, les ofrecen los medios para satisfacer sus metas personales, cuanto más comprendan y se les motive como operar estas exaltaciones, y cuanto más reflejen esta comprensión en el cumplimiento de sus acciones, mayores probabilidades habrá de que sean lideres eficientes. El líder retiene su condición de tal si: satisface necesidades resuelve problemas transforma, da un significado de existencia. 
Estrategia metodológica con el enfoque sociocomunitario productivo para el fortalecimiento de las habilidades de liderazgo en el proceso formativo de las estudiantes de la escuela Domingo Faustino de la localidad de Yura

Objetivo

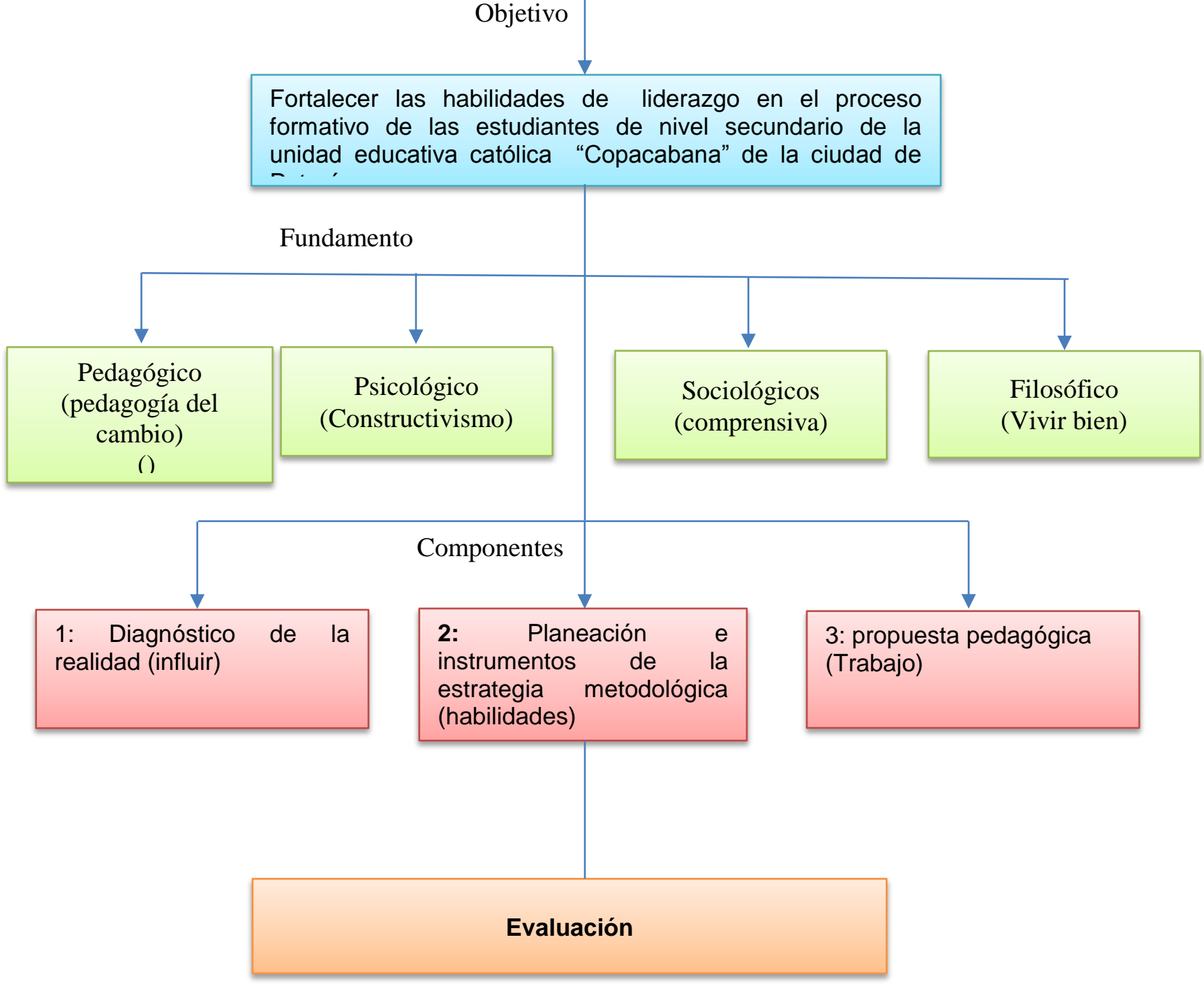

Figura 1. Propuesta pedagógica del liderazgo 


\section{Fundamento pedagógico}

Todos los pedagogos están de acuerdo que en la actualidad es necesario lograr un cambio en la Educación y para ello es necesario el cambio de ambiente pedagógico. En cuanto a la transformación de líderes en los estudiantes, a lo largo de nuestra vida profesional hemos sustentado una sola idea, un tanto compleja, pero una sola, que puede expresarse como sigue: El desarrollo de la institución educacional contemporánea se basa en la pedagogía del cambio, y se apoya en tres pilares fundamentales, que son: El desarrollo de directivos: como condición necesaria y resultado del desarrollo institucional; el trabajo en equipo como portador de creatividad, calidad y compromiso en las decisiones y las acciones y el liderazgo pedagógico, como la herramienta fundamental para el logro de los fines propuestos.

\section{Fundamento psicológico}

Hoy en día, y como consecuencia de un entendimiento más experimental al problema, se abre paso una nueva teoría acerca del liderazgo. Evidentemente, y aquí interesa subrayarlo, bajo esta concepción se implica la idea de que un mismo individuo puede mostrarse en realidad de diversas formas ante diferentes situaciones. Sin embargo, no sucede así en la niñez. Los chicos se entregan apasionadamente a la representación de "guardias y ladrones», mientras las chicas practican el papel de madres con sus muñecas. En las culturas primitivas es frecuente observar la utilización de estos juegos o representaciones como «procedimientos de enseñanza o aprendizaje». Incluso en nuestras sociedades más avanzadas se ha introducido el procedimiento para preparar entrevistadores y para adiestramiento de líderes. Por ello, se busca inclinar hacia a la psicología constructivista, donde se construye el conocimiento paso a paso de manera ascendente.

\section{Fundamento Filosófico}

Las escuelas tienen el reto de preparar y desarrollar a sus estudiantes en las competencias primordiales que permitan posteriormente a ese profesional desempeñarse como un líder creador de mejores formas de vivir y convivir en una sociedad en crisis. Tradicionalmente la educación universitaria se ha enfocado en el aprendizaje de técnicas que expanden el hacer. Por ello es necesaria la filosofía del vivir bien quien nos ayuda a comprender el fenómeno educativo de manera más amplia y racional.

\section{Fundamento sociológico}

La sociología ayuda a comprender al ser humano entendido como un ser social por excelencia, el hombre no puede estar solo; siempre necesita de los demás para poder desenvolverse y poder complementarse en su totalidad. Por ello, la sociedad es importante y es quien influye en el individuo debido a que la sociología intenta dar respuestas a las interrogantes que pueden surgir en relación al ser humano organizado en sociedad. Para ello se apuntara en esta propuesta en la sociología comprensiva de Max Weber quien ayuda a trabajar de manera efectiva y poder realizar cambios.

\section{Componentes}

1) Influir. Cuyo objetivo es intervenir en el discernimiento del liderazgo con el enfoque sociocumunitario productivo para ver el grado de conocimiento en los estudiantes de la unidad educativa Domingo Faustino Sarmiento en aplicación de los instrumentos del pre test y pos test. 
La aplicación fue concordada con la institución y se llevó a cabo con procesos de planificados pedagógicamente, respondió a una serie de instrumentos, que reflejaron el desarrollo de los mismos, en el marco de las variables objetivamente verificables, el objetivo del seguimiento, monitoreo $\mathrm{y}$ evaluación, fue establecer criterios cualitativos y cuantitativos de los componentes identificados, con el fin de establecer mejoras a corto, mediano y largo plazo, de esa manera asegurar la calidad, como variable perfectible de todo proceso en el marco de la formación continua.

**Pre test nos menciona el grado de confianza y las ganas de proseguir que tienen las/los estudiantes ante del inicio de las actividades o temáticas planificadas, para conocer con qué nivel de aprendizaje inicia el mismo estudiante individualmente. ¿Cómo se aplica el pre test? Su administración se desarrollara al inicio de cada temática, tomando de 1 a 10 minutos para su llenado, en la que los participantes otorgan una valoración de nunca, algunas veces, siempre según corresponda a cada una de las variables del instrumento.

**Pos test instrumentos que evaluara y aplicara a la finalización de las temáticas a realizar con los mismos indicadores para conocer el grado de aprendizaje de los estudiantes, realizado individualmente para conocer si el aprendizaje se desarrolló efectivamente. ¿Cómo se aplica el pos test? Su administración se desarrollara al finalizar la temática a las estudiantes, tomando de 1 a 10 minutos para su llenado, en la que los participantes otorgan una valoración según corresponda a cada una de las variables del instrumento. Este instrumento se llenara de la misma manera pero en diferentes momentos.

2) Habilidades. El proceso mediante el cual el estudiante va adquiriendo $\mathrm{y} / \mathrm{o}$ fortaleciendo competencias que le permiten tomar decisiones y realizar acciones para alcanzar sus propios objetivos y los del colectivo. Dicho proceso permite que la estudiante propicie cambios en lo social o en el comportamiento de su entorno, posicionándolo como un agente de cambio positivo para su contexto. Su objetivo es presentar la estrategia pedagógica para el desarrollo de las diferentes actividades en su estructura con la temática de estrategia metodológica con el enfoque sociocumunitario productivo.

3) Trabajo. Es necesario trabajar de manera conjunto con el director, los maestros y los escolares, para a través de ello lograr objetivos coherentes en el liderazgo. Su objetivo es presentar una propuesta de trabajo que vaya acorde a sus edades y capacidades para poder influir en ellos. Con las palabras claves: liderazgo, enfoque, trabajo, colaboración, cooperación, fortaleciendo las experiencias significativas de enseñanza/aprendizaje, que trasciendan y generen conocimiento de manera interdisciplinar, es el reto para construir y aprovechar a la vez las habilidades, competencias y motivación de los estudiantes en el momento de acompañar y orientar el proceso, alternado con la innovación de la práctica pedagógica, para aprovechar la relación dialógica entre el saber cotidiano y el saber científico, en la interacción docente/estudiante. La realización de las actividades se desarrollara con las estudiantes.

\section{Evaluación}

La evaluación se iniciara con la ayuda y valoración del profesor manifestando las dudas que tenga cada estudiante; la adquisición de motivación en aula, el de trabajar disfrutando un ambiente cálido en apoyo del educador al educando, con la cual se pueda tener resultados favorables en los 
temas principales para que él escolar tenga una clase diferente en el desarrollo de los contenidos profundizando las actividades $\mathrm{y}$ sea un buen líder.

\section{Discusión}

La formación es crecimiento personal y grupal. Favorece a todos y a cada uno de los miembros de la Comunidad Educativa. Se realiza a través de las estructuras organizativas. Es el que sirve de regulador para centrar cualquier análisis, debate o toma de decisión. Es una postura, una actitud ante la vida. Es tomar conciencia de que cualquier situación posibilita el crecimiento personal. Es tomar conciencia de que lo vivido en el centro repercute en otros ámbitos de la vida y viceversa. El crecimiento grupal se genera de la interacción que surge entre las personas. La implicación, la aportación personal potencia o frena este proceso.

Profesorado, alumnado y familias los acoge en su diversidad, en lo que cada persona es o manifiesta en una situación, ámbito o estructura determinada. Descartamos la idea de trabajar sólo con los que nos resultan afines, buscando la diversidad como fuente de riqueza. Es alrededor de la tarea marcada para cada estructura, donde se conforma el equipo. $Y$ es la tarea desde donde se reflexiona. Las estructuras se complementan, cada una abarca distintos ámbitos de funcionamiento del centro: aulas departamentos, paralelos, seminario completo, sector, ciclo. Una misma persona interviene desde distintas estructuras, es decir, trabajando con distintos equipos y sobre temáticas diferentes.

Para Aron, (1992) "Es una característica de sistema. No une elementos ajenos sin más, porque podría dejar de ser lo que es. Si partiendo de una necesidad personal, alguien se prepara leyendo o aprendiendo una teoría y la quiere aplicar directamente al sistema existente, eso sería unir desde fuera. $\mathrm{Si}$, por el contrario, esa teoría la interioriza, y desde ahí, con su determinada sensibilidad, aporta en cualquier estructura organizativa, forma parte del cuerpo cultural del centro. Esto es, generar desde dentro. Son los intereses del centro los que determinan los planes de formación, sin que esto vaya en detrimento de las opciones individuales que cada uno libremente, en función de sus posibilidades pueda desarrollar. Los desarrollos, lo que falta, lo que es requisito previo para conseguir lo siguiente, el tiempo con el que se cuenta".

Entre dichas disciplinas se alcanzó la humildad, confianza y creatividad, el líder debe ser así con un carácter determinante y una capacidad para lograr objetivos planteados en empresas, instituciones, diferentes áreas de vida y alcances satisfactorios. El líder debe empezar por encontrar la armonía consigo mismo y con su entorno, lograr primero un liderazgo personal, basado en sus principios a fin de dar una base firme a la planeación. También es necesario un conocimiento profundo de las propias posibilidades y de las de la competencia, para tratar de transformar las fuerzas de la competencia en beneficio propio. Y por último, la habilidad de seleccionar al personal adecuado y fomentar el trabajo en equipo; que se consigue siendo un líder que escucha, acepta ideas, admite sus errores, delega eficientemente, confía en sus colaboradores, mantiene la comunicación y el seguimiento entre equipos para logro de resultados (Valeria Pinto, 2010, p. 45).

Actualmente, la noción de formación de líderes suele ser asociada a la capacitación, por lo tanto, está vinculada a los estudios alcanzados y al aprendizaje que completó, ya sea a nivel formal con contenidos investigativos o informal el aprendizaje vivencial que día a día se va adquiriendo. Esto contempla dos ámbitos: por un lado, a la 
formación integral de la persona en relación con la comunidad y la Madre Tierra; y por otro, la integración de saberes y conocimientos técnico humanísticos. En ese sentido, la metodología a adoptar debe contribuir a dichos ámbitos y/o elementos.

En opinión de Méndez Benavides, (2008), no es que existan varios tipos de liderazgo: el liderazgo es uno y, como los líderes son personas (individuos con características personales definidas), las clasificaciones corresponden a la forma como ejercen o han adquirido la facultad de dirigir, circunstancia que no necesariamente implica que sea un líder. Existen tres tipos de liderazgo que se refieren a formas variadas de autoridad; líder Carismático, líder tradicional y líder legítimo.

La formación de líderes es tener conciencia, haber comprendido en el pensamiento y en la imaginación la educación del hombre antes que ella se realice. El hombre es el dueño de su destino. En nuestro medio, varios son los intelectuales de la educación que reconocen en la formación un devenir del Hombre; es decir, es autor de sí mismo. La formación conlleva una concepción sobre lo humano; sabernos no terminados y, en consecuencia, en necesidad de formación, de tomar forma. A cada ser humano le corresponde hacerse humano y, al lograrlo, continuar el proyecto de humanidad en el que está inscrito. En tal virtud, el ser humano es un ser no terminado. (Garzón-Valencia y Marín, 2013 p. 27).

Por ende, la formación debe permitir el pleno desarrollo de la persona, sus capacidades y competencias en el contexto social, político y económico. Por tal razón, la formación nos advierte la función de lo humano. Por ello formación es, la acción de "dar forma", y "forma" es lo que define algo como tal, lo que hace que algo sea eso y no otra cosa. Así, pues, la formación humana es la permanente construcción del ser de la persona, la manera particular de ser sí mismo. Dicha construcción, es un proceso que se genera y se dinamiza a través de acciones orientadas hacia la transformación de los sujetos; por ello afirma cambiar que "las actividades de formación hacen Profesorado. (Pini, 2002, p. 48).

Según River, (2009) el Liderazgo es el conjunto de habilidades gerenciales $o$ directivas que un individuo tiene para influir en la forma de ser de las personas o en un grupo de personas determinado, haciendo que este equipo trabaje con entusiasmo, en el logro de metas y objetivos. También se entiende como la capacidad de tomar la iniciativa, gestionar, convocar, promover, incentivar, motivar y evaluar a un grupo o equipo. En la administración de empresas el liderazgo es el ejercicio de la actividad ejecutiva en un proyecto, de forma eficaz y eficiente.

Considerando la complejidad real en el desarrollo y ejercicio de conocimientos $\mathrm{y}$ habilidades de liderazgo, es que actualmente se propone tanto el diseño de una sucesión planificada, con etapas progresivas de experimentación y certificación, como el fomento del liderazgo distribuido, tanto a nivel de sistema como al interior de las escuelas (Galdames, 2010, pp. 27-28).

El tema de si el líder debe ser el mejor líder, es un aspecto que ha dado mucho que hablar. Mi punto de vista es que el líder no siempre debe ser un experto en la materia, quizás en su equipo hayan personas más expertas en uno u otro campo que él o ella. Hay algunas empresas que promocionan como líder, llegando a cumplir en ocasiones con el principio de Peter en el que "una persona acaba ascendiendo hasta su máximo nivel de incompetencia”. (Pini, 2002, p. 23).

Como se ha dicho la estrategia es un procedimiento heurístico que permite tomar decisiones en condiciones específicas. Una 
estrategia es un conjunto finito de acciones no estrictamente secuenciadas que conllevan un cierto grado de libertad y cuya ejecución no garantiza la consecución de un resultado óptimo; por ejemplo, planificar una entrevista, llevar a cabo una negociación, la orientación topográfica, resolución de problemas, realizar un cálculo mental, planificación de una excursión por una montaña desconocida, ejecutar una decisión adoptada, etc.

Ahora bien, existen mil y una maneras de aprender pero, llama la atención la uniformidad en la práctica pedagógica y la poca variedad de técnicas metodológicas utilizadas. Para muchos profesores con tener una pizarra y la tiza les basta. De esta manera se corre el riesgo de aburrir a los alumnos y de aburrirse el profesor mismo (Quinquer, 2004, p. 22).

Una técnica metodológica es un procedimiento algorítmico; es una manera de hacer algo que se aplica a una actividad determinada. La técnica metodológica es un método específico, es la forma concreta de aplicar un método y supone una organización de las actividades en el aula por parte del profesor y la utilización de los materiales didácticos, sean audiovisuales, máquinas, libros, apuntes, etc. Es la forma concreta como el estudiante o un grupo de estudiantes aplica un método de aprendizaje al realizar una actividad, a fin de desarrollar destrezas y actitudes. Cada uno de estos métodos puede ser utilizado por el profesor en ciertos momentos. (Pozo, 2013, p. 17).

\section{CONCLUSIONES}

Coexisten diversas conceptualizaciones de liderazgo pero todos coinciden en inspirar confianza y coadyuvar en el logro de objetivos, así como también el trabajo en conjunto con su gente, por ello es importante conocer el nivel de los procesos de enseñanza para tomar decisiones ya que en el fondo se trata de crear las condiciones para que estas nuevas generaciones $\mathrm{y}$ en particular los escolares puedan para construir su mundo.

La propuesta realizada promoverá el aprendizaje colaborativo y aportará las herramientas para que los estudiantes adquieran el abordaje del tema tanto en la parte teórica como en la práctica, obedeciendo así los indicadores del liderazgo. Ciertamente se ocupa demasiado tiempo en aquellas acciones que se llevaran a cabo para el conocimiento del docente y estudiante. Sin embargo, vale la pena porque en la actualidad la responsabilidad social no es únicamente generar economía o policía, sino que nos lleva a proveer colaborativamente estrategias para el aprendizaje de los estudiantes.

El proceso educativo de liderazgo es de gran importancia, por ello se recomienda dar seguimiento a la implementación, para que en un estudio a futuro se pueda dar temáticas en cuanto a las acciones investigativas del líder.

Es recomendable indagar en la Ley 070, debido a que en Bolivia se trabaja con esta Ley. Ella plantea la transformación para los estudiantes, y considera de vital importancia el desarrollo de habilidades sociales, entendida como iniciativas $\mathrm{y}$ respuestas efectivas y apropiadas, las cuales influyen en el comportamiento verbal y no verbal, que no vienen determinadas de forma innata, al contrario son capacidades que se adquieren dando lugar a mejorar las relaciones sociales, ello es una búsqueda de convivencia positiva entre los estudiantes.

Así los proceso educativos estarán inspirados en una visión para lograr mejorar resultados y mejores niveles de aprendizaje y eso se lograra con ayuda de estrategias para la implementación en cada aula de aprendizaje. Del mismo modo se recomienda implementar en unidades educativas estrategias metodológicas para impartir una 
educación constructiva y liberal, lo que conllevará a mejorar su competitividad y a fomentar en los estudiantes el liderazgo que es uno de los factores primordiales para el desarrollo y cambio de las personalidades educativas y para la transformación de todos.

\section{REFERENCIAS}

Aron, R. (1992). Dimensiones de la Conciencia Histórica. 2a Editorial. México: Fondo de Cultura Económica

Coleman, D. (2012). Que define el lider metodólogicas. New ork: Times

Galdames, S., y Espinoza, S. R. (2010). Líderes educativos previo a cargos directivos. Una nueva etapa de formación. REICE: Revista Iberoamericana sobre Calidad, Eficacia y Cambio en Educación, 8(4), 5064

Garzón Valencia, S. M., y Marín Carvajal, D. A. (2013). Caracterización del estilo de liderazgo y la percepción de sus colaboradores (Caso Aguas de Manizales). Méndez Benavides, J. R. (2008). Teoría del Liderazgo. Costa Rica: Ey-Blanchard

Pini, F. R. (2002). Formación de Lideres y Movimientos Sociales (Experiencias y Propuestas Educativas). Ecuador: Abya Yala

Pozo, C. J. (2013). Metodologia estrategiasy tecnicas. Lima: Champagnat

Quinquer, D. (2004). Estrategias metodológicas para enseñar y aprender ciencias sociales: interacción, cooperación y participación. Barcelona: Íber

River, R. A. (2009). Liderazgo. Mexico: Castillo Rojas, A., y Gaspar, F. (2006). Bases del liderazgo en educación. Orealc/Unesco.

Sampieri, P. D. (2010 ). Metodologia de la investigación. Guatemala: McGrawHill

Valeria Pinto, S. G. (2010). psicoperspectivas, individuo y sociedad. en s. g. valeria pinto, psicoperspectivas, individuo y sociedad (pág. 157). chile: children 